\title{
A segurança do trabalhador de enfermagem na administração de quimioterápicos antineoplásicos por via endovenosa
}

\author{
Nursing workers' safety during intravenous administration of antineoplastic \\ chemotherapeutics

\section{Seguridad de los trabajadores de enfermería en la administración de quimioterápicos antineoplásicos por vía intravenosa}

\author{
Monique Haenscke SennaI; Cândida Custódio da Silva ${ }^{\text {II }}$ Francine Lima Gelbcke ${ }^{\text {III; }}$ \\ Jane Cristina Anders ${ }^{I V}$; Maria Patrícia Locks de MesquitaV
}

\begin{abstract}
RESUMO: A exposição ocupacional dos trabalhadores que manipulam drogas quimioterápicas antineoplásicas pode ocorrer durante o preparo, administração e seu descarte, sendo imprescindível o uso correto de equipamentos de proteção individual (EPIs). Estudo de natureza qualiquantitativa, realizado no sul do Brasil. Objetivou-se identificar o conhecimento dos trabalhadores acerca do uso correto dos EPI's, e analisar o processo de administração dos agentes químicos, pelos trabalhadores de enfermagem, em unidade de clínica médica e ambulatório de quimioterapia de um hospital universitário. Na coleta de dados, em 2010, foram utilizados questionário e observação de campo. Identificou-se que os trabalhadores conhecem os cuidados de proteção, mas não os realizam efetivamente na unidade de internação, diferente do observado no ambulatório, onde o serviço é especializado no manuseio de tais drogas, gerando um comprometimento maior com a sua segurança. Os resultados revelaram a necessidade de educação permanente para conscientização do risco de exposição às drogas antineoplásicas.
\end{abstract}

Palavras-Chave: Saúde do trabalhador; riscos ocupacionais; quimioterapia; equipamentos de proteção.

\begin{abstract}
Occupational exposure of workers handling antineoplastic chemotherapy drugs may occur during preparation, administration and disposal, and correct use of personal protective equipment (PPE) is essential. This qualitative and quantitative study was conducted in southern Brazil in order to identify workers' knowledge of proper use of PPE, and to analyze the process of administration of these chemical agents by nursing staff on an internal medicine ward and an outpatient chemotherapy unit at a university hospital. Data were collected in 2010 by questionnaire and field observation. It was ascertained that workers knew what protection precautions to take, but did not actually taken them on the ward, unlike what was seen at the outpatient clinic, where the service specializes in handling these drugs, leading to greater commitment to security. The results revealed the need for continuing education to build awareness of the risk of exposure to antineoplastic drugs. Keywords: Workers' health; occupational hazards; chemotherapy; protective equipment.
\end{abstract}

RESUMEN: La exposición ocupacional de trabajadores que manipulan fármacos antineoplásicos puede acaecer durante la preparación, administración y descarte de los medicamentos, siendo esencial el correcto uso de equipos de seguridad (ESs). Estudio de carácter cualitativo y cuantitativo, realizado en el sur de Brasil. Su objectivo fue identificar el conocimiento de trabajadores sobre el uso correcto de ESs y analizar el proceso de administración de los fármacos, en unidad clínica y unidad de quimioterapia ambulatorial de un hospital universitario. En la recolección de datos, en 2010, fueron utilizados cuestionario y observación de campo. Se identificó que los trabajadores conocen los cuidados de protección, pero no los realizan con eficacia en la unidad de hospitalización, diferente del observado en ambulatorio, donde el servicio es especializado en el manoseo de eses fármacos, generando mayor compromiso con su seguridad. Los resultados revelaron la necesidad de educación contínua para el conocimiento de la exposición al riesgo de los fármacos antineoplásicos.

Palabras Clave: Salud del trabajador; riesgos laborales; quimioterapia; equipo de protección.

\section{INTRODUÇÃO}

A saúde ocupacional reflete uma resposta institucional aos movimentos sociais que, entre a metade dos anos 70 e os anos 90, reivindicaram que as questões de saúde relacionadas ao trabalho fizessem parte do direito universal à saúde, sendo incluídas no escopo da saúde pública1. A saúde do trabalhador no Brasil

\footnotetext{
IEnfermeira. Doutoranda do Programa de Pós-Graduação em Enfermagem da Universidade Federal de Santa Catarina. Membro do Grupo de Estudos e Pesquisas em Administração e Gerência do Cuidado em Enfermagem e Saúde. Membro do Grupo de Estudos e Pesquisas em Administração e Gerência do Cuidado em Enfermagem e Saúde. Florianópolis, Santa Catarina, Brasil. E-mail: moniquehsenna@hotmail.com.

"Enfermeira. Especialista em Saúde da Família. Florianópolis, Santa Catarina, Brasil. E-mail: candycustodio@yahoo.com.br.

IIIEnfermeira. Doutora em Enfermagem. Professora do Departamento de Enfermagem, do Programa de Pós-Graduação de Enfermagem e do Mestrado Profissional em Gestão do Cuidado em Enfermagem da Universidade Federal de Santa Catarina. Integrante do Grupo de Estudos sobre Trabalho, Cidadania, Saúde e Enfermagem. Florianópolis, Santa Catarina, Brasil. E-mail: francine.lima@ufsc.br.

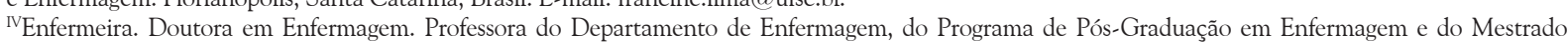
Profissional em Gestão do Cuidado em Enfermagem da Universidade Federal de Santa Catarina. Líder do Grupo de Ensino, Pesquisa e Extensão na Saúde da Criança e do Adolescente. Florianópolis, Santa Catarina, Brasil.E-mail: jane.anders@ufsc.br.

vEnfermeira do Hospital Universitário. Doutoranda do Pós-Graduação em Enfermagem da Universidade Federal de Santa Catarina. Membro do Grupo Práxis. Florianópolis, Santa Catarina, Brasil. E-mail: pattylocks@yahoo.com.br.
} 
surge durante o período de transição democrática, onde se iniciam os questionamentos acerca da interferência do trabalho no processo saúde-doença. Esse momento é caracterizado pela existência de epidemias, doenças profissionais clássicas e o surgimento de novos adoecimentos ocasionados no ambiente de trabalho, decorrentes da globalização e mudanças nas práticas laborais ${ }^{2-5}$. O seu bem-estar físico, mental e social está intimamente relacionado a fatores sociais, econômicos, organizacionais e tecnológicos.

Os trabalhadores estão constantemente expostos a danos quando em contato com diferentes riscos ocupacionais: físico, ergonômico, químico, biológico e psicossocial, cuja importância está relacionada à categoria profissional e à área de atuação ${ }^{3}$. O reconhecimento do risco a que o profissional está exposto consiste em prever situações ou eventos em seu ambiente de trabalho que poderão ocasionar lesões corporais ou perturbação funcional, baseado em conhecimento prévio ou estimado, com o objetivo de adotar condutas e precauções padrões para minimizar os riscos ${ }^{4}$. Para proteção do trabalhador, durante o manuseio de quimioterápicos, é imprescindível a utilização de equipamentos de proteção individual (EPI's), tais como luva não entalcada, aventais descartáveis com punho elástico e máscaras com filtro de carvão ativado $^{3-5}$.

Nesse sentido, com este estudo busca-se responder à seguinte questão norteadora: Como são implementadas as normas de segurança do trabalhador na administração de quimioterápicos antineoplásicos (Qt-An) endovenosos, em um hospital universitário.

Foram estabelecidos como objetivos: identificar o conhecimento dos trabalhadores acerca do uso correto dos EPI's e analisar o processo de administração dos citados agentes químicos, pelos trabalhadores de enfermagem, em uma unidade de clínica médica e ambulatório de quimioterapia de um hospital universitário.

\section{REVISÃO DE LITERATURA}

A incidência do câncer cresce no Brasil, como em todo o mundo, num ritmo que acompanha o envelhecimento populacional decorrente do aumento da expectativa de vida. É o resultado direto das grandes transformações globais das últimas décadas, que alteraram a situação de saúde dos povos pela urbanização acelerada, dos novos modos de vida e novos padrões de consumo.

Câncer ocupacional é ocasionado pela exposição, durante a vida laboral, a agentes cancerígenos presentes nos ambientes de trabalho. Os fatores de risco podem ser ambientais ou hereditários, podendo ambos estar relacionados. Esses fatores interagem de várias formas para dar início às alterações celulares envolvidas na etiologia do câncer. Segundo alguns estudos, em média, 2\% a $4 \%$ de todos os casos de câncer podem estar associados a exposições ocorridas nos ambientes de trabalho ${ }^{6-8}$.

Os hospitais apresentam uma variedade de riscos, dentre os quais os agentes químicos parecem estar relacionados mais intensamente com as drogas antineoplásicas, os gases anestésicos e os agentes esterilizantes. É notório que a exposição a Qt-An traz benefício aos pacientes, porém, os riscos aos profissionais, que estão continuamente expostos a esses agentes, devem ser avaliados. Esses agentes químicos podem provocar nos trabalhadores intoxicações agudas, estados crônicos e pré-patológicos de várias doenças ocupacionais?

A exposição ocupacional aos trabalhadores de saúde que manipulam as drogas utilizadas na quimioterapia antineoplásica, pode ocorrer em qualquer das três fases de contato com estas substâncias: preparo, administração e descarte dos quimioterápicos antineoplásicos. Durante o preparo, os riscos de exposição podem acontecer durante a abertura de ampolas, na reconstituição das drogas, na retirada de solução do frasco-ampola e na retirada de ar da seringa que contém antineoplásicos. Em relação ao descarte, o risco está presente quando os trabalhadores não adotam as medidas de biossegurança durante o manuseio de fluidos corpóreos, desprezo de materiais que entraram em contato com fluidos corpóreos e manipulação de roupas contaminadas por estes fluidos, bem como mediante o acondicionamento incorreto de materiais contaminados. Ou seja, a contaminação por quimioterápicos antineoplásicos ocorre da exposição direta ou indireta a estas substâncias ${ }^{8}$.

Para proteger o trabalhador durante o manuseio de quimioterápicos e de excretas de pacientes submetidos à quimioterapia, é considerada essencial a adoção de medidas, como a utilização de câmaras de fluxo laminar vertical para o preparo de antineoplásicos e o uso de EPI corretamente, nas diversas atividades que envolvem a manipulação de quimioterápicos.

\section{Metodologia}

O presente estudo descrito é de natureza quantitativa. Os dados foram obtidos através da aplicação de um questionário estruturado com questões de caráter múltiplo, questões abertas e fechadas, e um instrumento de observação focal, de cunho não participativo. Foram cumpridas as determinações da Resolução no 196 de 1996 do Conselho Nacional de Saúde, que diz respeito aos aspectos éticos de pesquisa com seres humanos, sob a aprovação do Comitê de Ética em Pesquisas com Seres Humanos (CEPSH), de Certificação $n^{\circ} 918$. Os profissionais de enfermagem que participaram deste estudo assinaram o Termo de Consentimento Livre Esclarecido. 
O estudo foi realizado num hospital escola da Região Sul do Brasil, sendo escolhida para desenvolver o estudo a unidade de clínica médica II, em que ficam internados os pacientes da hematologia que fazem uso de quimioterápicos e a unidade de quimioterapia ambulatorial.

Os participantes do estudo foram os profissionais que compõem a equipe de enfermagem (enfermeiro, técnico de enfermagem e o auxiliar de enfermagem) de ambos setores, no período de agosto a novembro de 2010. Para garantir o sigilo e o anonimato dos participantes, os trabalhadores fora identificados pelas letras E para enfermeiro, T para técnico de enfermagem e A para auxiliar de enfermagem, seguidas de numeração crescente.

Responderam ao questionário da pesquisa 20 profissionais de enfermagem, sendo 6 enfermeiros, dos quais 1 do ambulatório de quimioterapia e 5 da unidade de internação médica; 10 técnicos de enfermagem, sendo 2 do ambulatório de quimioterapia e 8 da unidade de internação médica; e 4 auxiliares de enfermagem, sendo 1 do ambulatório de quimioterapia e 3 da unidade de internação médica.

$\mathrm{Na}$ observação de campo, foram acompanhados 10 trabalhadores de enfermagem durante a administração de quimioterápicos: 1 enfermeiro do ambulatório de quimioterapia; 7 técnicos de enfermagem, sendo 2 do ambulatório de quimioterapia e 5 da unidade de internação médica; e 2 auxiliares de enfermagem, sendo 1 do ambulatório de quimioterapia e outro da unidade de internação médica, viabilizando, assim, o segundo instrumento da pesquisa, a observação de campo.

A análise dos dados qualitativos permitiu seu agrupamento por similaridade, possibilitando a sua interpretação. Já, em relação à abordagem quantitativa, foi realizada análise estatística, com cálculos de frequência absoluta e relativa.

\section{Resultados e Discussão}

Em função da rotatividade de pacientes e especificidades do tratamento quimioterápico, não foi possível realizar concomitantemente a aplicação dos questionários e o preenchimento do instrumento de observação. Assim, primeiramente aplicou-se o questionário e, após, conseguiu-se observá-los, em campo.

No questionário de campo, foram levantados dados referentes ao sexo, categoria profissional, unidade em que trabalha, tempo de formação e de trabalho na instituição, turno de trabalho, ou seja, dados que possibilitassem traçar um perfil da amostra; e dados relativos ao objeto do estudo - conhecimento e utilização de EPI's em Qt-Na; como: finalidade da quimioterapia; importância da utilização de EPI's na administração de quimioterápicos e EPI's utilizados; riscos a que estão expostos quando da aplicação de Qt-Na; sintomas subsequentes à aplicação; e se haviam sofrido algum acidente relacionado à administração de Qt-An. Caso respondessem que já tinham sofrido acidente com QtAn, era ainda questionado: como ocorreu o acidente, qual a sintomatologia apresentada, que providências foram tomadas e se havia conhecimento dos efeitos colaterais decorrentes do acidente. Buscou-se, dessa forma, confrontar os dados dos questionários aos da observação em campo, pois, muitas vezes, o trabalhador sabe dos cuidados necessários, porém não os utiliza na prática cotidiana.

Na observação de campo, foram acompanhados sete técnicos de enfermagem, dois auxiliares de enfermagem e um enfermeiro. Estes números têm relação com a composição da força de trabalho, na qual há expressivo número de trabalhadores de enfermagem de nível médio, e com as atividades definidas pela lei do exercício profissional, que determina aos profissionais enfermeiros a supervisão dos serviços e a assistência direta, principalmente, aos pacientes graves. Quanto à força de trabalho, nas estatísticas de profissionais cadastrados para o Estado de Santa Catarina, no ano de 2010, 33.700 (80\%) dos profissionais são de nível médio, sendo 23.784 (56\%) técnicos de enfermagem e 9.916 (24\%) auxiliares de enfermagem, 8.422 (20\%) são profissionais de nível superior (enfermeiros) ${ }^{9}$. Este quantitativo menor de enfermeiros nas instituições de saúde pode estar influenciando as atividades realizadas por estes profissionais, no que se refere à assistência direta. Há que se ressaltar que só foi observado o profissional enfermeiro administrar esse tipo de droga no ambulatório de quimioterapia, sendo que na unidade de internação este cuidado não tem sido realizado pelo enfermeiro. Pode-se supor que esse cuidado realizado em nível ambulatorial se deve ao fato de existir um menor quantitativo de profissionais nesse serviço, associado à maior demanda de administração de Qt-An.

Também foram levantados dados referentes a conhecimentos gerais acerca da quimioterapia, como finalidade, risco de exposição, quais EPI's são necessários na administração de Qt-An, entre outros. Nas respostas dos participantes, 16 (80\%) trabalhadores assinalaram que a finalidade da quimioterapia é o tratamento de câncer, 15 (75\%) apontaram a destruição de células cancerígenas, 13 (65\%) - impedir, controlar ou reduzir o crescimento tumoral, 11(55\%) - evitar o aparecimento de metástase e 6 (30\%) - redução de gânglios. De maneira geral, os trabalhadores conhecem o objetivo do tratamento quimioterápico, porém de maneira superficial e limitada, sugerindo déficit de educação permanente e aprofundamento do tema.

Quanto ao uso dos EPIs, os dados apresentados referem-se ao que foi levantado nos questionários e na observação de campo, conforme apresentado na Tabela 1. 
TABELA 1: EPI's necessários na administração de Qt-An, a partir do questionário e da observação de campo. Florianópolis -SC, 2010.

\begin{tabular}{lcccc}
\hline \multirow{2}{*}{ EPI's } & \multicolumn{3}{c}{ Questionário } & \multicolumn{2}{c}{ Observação } \\
\cline { 2 - 5 } & $\mathrm{f}$ & $\%$ & $\mathrm{f}$ & $\%$ \\
\hline Avental fechado atrás, de manga longa, punho & 18 & 90 & - & - \\
elástico e descartável & 18 & 90 & 3 & 30 \\
Máscara com filtro de carvão ativado & 18 & 90 & 1 & 10 \\
Óculos de proteção & 17 & 85 & 5 & 50 \\
Luva grossa de látex, longa e não entalcada & 7 & 35 & 8 & 80 \\
Avental de tecido, fechado frontalmente & 6 & 30 & 6 & 60 \\
Luva de procedimento & 4 & 20 & - & 0 \\
Máscara bico de pato & 4 & 20 & 1 & 10 \\
Touca/gorro & 3 & 15 & 1 & 10 \\
Propé & 3 & 15 & - & - \\
Máscara cirúrgica & 2 & 10 & - & - \\
Luva estéril & 1 & 5 & 1 & 10 \\
Macacão polietileno & - & - & 2 & 20 \\
Luva plástica transparente & - & - & 2 & 20 \\
Máscara comum & & & &
\end{tabular}

Ao analisar os dados da observação e confrontar com os questionários, notou-se uma significativa divergência entre a realidade observada e a realidade descrita, especialmente no uso de EPI's, elemento fundamental para a biossegurança individual, coletiva e ambiental. A maioria dos participantes descreveu fazer uso de equipamentos além do preconizado, segundo dados da Tabela 1, referentes ao questionário, a fim de uma cobertura maior de proteção; porém constatou-se a negligência desses profissionais a frente à exposição a substâncias antineoplásicas, conforme dados da Tabela 1, referentes à observação.

As normas da Agência Norte-Americana Occupational Safetyand Health Administration (OSHA) estabelecem como obrigatório o uso de EPI's para a manipulação e administração de antineoplásicos e especificam a utilização de aventais de mangas longas, punhos elásticos, com frente fechada e descartável; óculos de proteção total; máscaras com proteção de carvão ativado; e luvas grossas de prolipropileno ou látex, não entalcadas e descartáveis ${ }^{5}$. As recomendações das precauções padrão vêm sendo utilizadas pelos profissionais da saúde há mais de 15 anos, principalmente após a adesão das precauções universais de proteção. Essa prevenção primária continua a ser eficaz e eficiente na proteção dos trabalhadores diante de riscos ocupacionais ${ }^{10}$. $\mathrm{O}$ recomendável é que o profissional esteja sempre protegido, durante a assistência aos pacientes, fazendo uso constante dos EPI's. As maiores causas de acidentes entre os trabalhadores da equipe de enfermagem acontecem durante o manuseio de objetos perfurocortantes em função da falta de adesão ao uso de EPI's ${ }^{8}$.

Ressalta-se que a instituição, de uma forma geral, oferece tais equipamentos de proteção para uso dos trabalhadores, o que não justifica, portanto, a não utilização dos mesmos. Uma ação que poderia modificar esta falta de adesão aos EPI's seria a educação permanente desses profissionais, realizando oficinais de estudos e disponibilizando materiais informativos, a fim de despertar-lhes a atenção para os riscos que correm ao entrar em contato direto com as substâncias antineoplásicas. Para que isso aconteça, há de se reorganizar o processo de trabalho no serviço, onde já está havendo discussão sobre o tema na instituição de estudo.

Quanto às demais medidas necessárias, a instituição procura seguir o preconizado pela Agência Nacional de Vigilância Sanitária (ANVISA) e demais órgãos específicos, assegurando o fornecimento de equipamentos de proteção individual e coletiva, uso de capela de fluxo laminar vertical para a manipulação e caixas específicas para o descarte de material tóxico ${ }^{11}$, porém há certa resistência ao uso correto e completo desse material, evidenciando a necessidade de educação permanente.

No levantamento da importância da utilização de EPI's, constatou-se o limitado conhecimento acerca do real valor desse material, não só para a proteção individual, mas também para a proteção coletiva, uma vez que se almeja a segurança própria e do outro, dentro de um contexto ético. Destacaram-se as respostas dos questionários:

É fundamental o profissional ter consciência em relação ao uso de EPI como também em relação à cultura de segurança existente na instituição. É responsabilidade do profissional o uso adequado de EPI para cada situação e responsabilidade da instituição oferecer estes equipamentos. (E16)

Reduzir ao máximo as possibilidades de contaminação por acidentes com os medicamentos utilizados tanto EV [endovenoso] como os VO [via oral]. (T17) 
Em relação aos riscos a que estão expostos no contato com o quimioterápico, os dados apresentados referem-se ao que foi levantado nos questionários, conforme ordenados na Tabela 2 .

Observou-se que os trabalhadores de enfermagem conhecem os riscos de exposição à Qt-An em curto prazo, ou seja, ao entrarem em contato direto com a droga ou a ocorrência de acidentes de trabalho, porém a maioria desconhece os efeitos a longo prazo, conforme mostra a Tabela 2. Todavia, informações científicas alertam que vários são os sintomas que ocorrem com trabalhadores que manipulam Qt-An, entre os quais se destacam os efeitos imediatos, como tontura, cefaleia, náuseas, alterações de mucosas e reações alérgicas, entre outros ${ }^{12}$.

Logo, cuidados relacionados aos profissionais que manuseiam os antineoplásicos são necessários devido ao potencial mutagênico das medicações, exigindo o seguimento de normas técnicas e de segurança para a manipulação desses agentes ${ }^{6}$. Constitui-se papel fundamental do enfermeiro a supervisão da equipe de enfermagem, alertando esses profissionais sobre os graves riscos a que estão expostos quando não protegidos adequadamente durante a manipulação de quimioterápicos.

Sobre os efeitos adversos da administração de quimioterapia, 9(45\%) responderam não sentir nada, $8(40 \%)$ referiram cefaléia e 4(20\%)-náuseas, $3(15 \%)$-irritação na garganta e $1(5 \%)$-calor na face, efeitos estes identificados na literatura ${ }^{12}$. Dos 20 participantes que responderam aos questionários, 5(20\%) relataram ter tido acidentes com a administração de quimioterapia.
No setor de quimioterapia (local de infusão), ao instalar no paciente, houve vazamento do mesmo no meu jaleco e no chão. (T18)

Houve rompimento na conexão de borracha do equipamento na I, ocasionando o extravasamento da quimioterapia no meu rosto. O acidente ocorreu em um plantão diumo na CM II/HU aproximadamente há 1 ano. (A13)

Os trabalhadores que sofreram acidente, com vazamento de Qt-An, relataram sentir sintomas semelhantes às reações adversas que o paciente apresenta durante e após o tratamento, como:

Cefaléia e irritação na garganta. (E5)

Alterações da mucosa oral e cefaleia. (E16)

Sobre as providências assumidas após o acidente, foram referidas medidas de proteção coletiva, pensando e focando mais no próximo-colegas de profissão, pacientes e familiares/acompanhantes - do que em si mesmo, como descrito a seguir:

Proteção do local, descarte de roupas de cama, cuidados com o paciente. Em relação a mim, nada. (E5)

Ressalta-se que a instituição tem protocolo de extravasamento de quimioterápicos e o suporte da Divisão de Saúde e Segurança do Trabalho com o intuito de promover a saúde e assegurar condições adequadas ao trabalhador/servidor, fornecendo formulários de comunicação de acidentes e comunicação de riscos no trabalho ${ }^{13}$. A resposta, seguinte, demonstra os cuidados no caso de acidentes, quer com os pacientes, quer com os trabalhadores:

Utilização do protocolo de extravasamento, encaminhamento de todo o material utilizado para a limpeza

TABELA 2: Resposta dos questionários acerca dos riscos da exposição à Qt-An. Florianópolis-SC, 2010

\begin{tabular}{lcc}
\hline \multicolumn{1}{c}{ Itens dos questionários } & \multicolumn{2}{c}{ Respostas } \\
\cline { 2 - 3 } & $\mathrm{f}$ & $\%$ \\
\hline Inalação da droga & 20 & 100 \\
Contato direto de quimioterápicos com a pele e mucosas & 19 & 95 \\
Ocorrência de lesão tecidual & 17 & 85 \\
Ocorrência de acidentes & 16 & 80 \\
Ocorrência de reações alérgicas & 15 & 75 \\
Infertilidade & 11 & 55 \\
Mutagenicidade & 8 & 40 \\
Ocorrência de imunodepressão & 8 & 40 \\
Desenvolvimento de câncer & 5 & 25 \\
Disfunções menstruais & 4 & 20 \\
Ocorrência de anemia & 3 & 15 \\
Queda de cabelos e pelos & 2 & 10 \\
Queda de cabelo & 1 & 5 \\
Desconforto gástrico & 1 & 5 \\
Cefaléia & 1 & 5 \\
Alteração visual & 1 & 5 \\
\hline
\end{tabular}


do recipiente de resíduo químico. Comunicar e realizar registro do acidente junto ao serviço de segurança do trabalho e solicitação de nova contagem de partículas no ambiente. (E16)

Quanto aos efeitos colaterais, tanto a longo prazo - após exposição a agentes antineoplásicos, como a curto prazo-logo após acidente, os profissionais evidenciaram ter conhecimento dos riscos, porém, sem atribuir relevância a esta questão:

A longo prazo pode trazer alterações celulares, porém acredito que um episódio isolado não trará consequências. (E5)

Os efeitos da exposição a agentes químicos são cumulativos e somados ao estilo de vida, onde fatores ambientais tendem a potencializar os efeitos colaterais e as consequências à saúde do indivíduo ${ }^{14}$. A atuação do enfermeiro na conscientização do profissional torna-se determinante ao orientar este trabalhador a adotar medidas de biossegurança capazes de prevenir danos possivelmente irreversíveis.

O cuidado prestado no ambulatório de quimioterapia é mais direto e focal, uma vez que o paciente fica de $1 \mathrm{~h}$ a $4 \mathrm{~h} 30 \mathrm{~min}$ no serviço para o tratamento. Vale ressaltar que o espaço físico do setor apresentase em formato de ilha, facilitando o monitoramento do paciente e das possíveis reações adversas. Em função de o ambulatório ter capacidade para receber sete pacientes ao mesmo tempo para infusões de QtAn, notou-se maior preocupação dos funcionários em relação à contaminação do ambiente, ou seja, ao uso de ventilação, ao cuidado minucioso para a instalação da infusão e o descarte de todo o material utilizado. Observou-se que em todas as circunstâncias os profissionais utilizavam, no mínimo, luvas de procedimentos.

Já, na clinica médica, o paciente recebe Qt-An em push e infusão. Como, no setor, os funcionários além de cuidar dos pacientes oncológicos, cuidam de pacientes de outras especialidades, uma vez que são 29 leitos na unidade de internação, o cuidado é realizado de forma generalizada, tornando-se muitas vezes inviável um cuidado focal. Observou-se que o cuidado ao longo da infusão (desde a instalação, à manipulação das excretas e ao descarte do material utilizado) não é realizado com a devida atenção, sugerindo, assim, estarem manipulando um medicamento qualquer, sem, muitas vezes, EPI's.

Vale ressaltar a importância do enfermeiro na vigilância, promoção da saúde e segurança do trabalhador de saúde, especialmente, o trabalhador que lida com quimioterápicos. As ações voltadas para a biossegurança devem ser continuadamente desenvolvidas pela equipe de enfermagem, principalmente, por prestar assistência direta ao paciente, estando constantemente exposta aos riscos próprio dessa atividade $^{15}$. $O$ enfermeiro deve supervisionar sua equipe e promover ações de educação permanente, a fim de excluir possíveis riscos ocupacionais aos trabalhadores expostos a drogas quimioterápicas.

Supervisionar, orientar e alertar a equipe de enfermagem é uma função primordial do enfermeiro como líder desse grupo. O cuidado com a saúde dos trabalhadores pode ser reforçado através da educação continuada, oficinas educativas, materiais informativos e, principalmente, com participação ativa do enfermeiro nos cuidados prestados aos pacientes oncológicos. A equipe precisa estar ciente da possível intervenção do enfermeiro em repreender o profissional, no caso de negligência, pois esta medida certifica um cuidado seguro tanto para a saúde do paciente quanto a do profissional.

\section{ConClusão}

É crescente o uso de quimioterápicos antineoplásicos como uma das formas de tratamento de câncer. Na mesma proporção, o índice de exposição ocupacional a esses fármacos acompanha o seu uso, apontando um olhar mais apurado para os riscos que essa exposição pode acarretar à saúde do trabalhador, ao longo de sua vida.

Há evidências científicas de que os efeitos colaterais sentidos pelos pacientes, durante o tratamento, também são relatados pelos profissionais, sendo mais descritos desconfortos como cefaléia, mucosite e reações alérgicas. Já, em estágio mais avançado, há evidências de risco carcinogênico associado a hábitos alimentares inadequados, atividade física inexistente, uso indevido de substâncias, predisposição genética, bem como histórico familiar da doença.

Da mesma forma, é unanimidade a biossegurança do paciente frente a agentes nocivos. Faz-se necessária a segurança do trabalhador, compreendendo, assim, a segurança individual, coletiva e do ambiente, respeitando-se princípios éticos e direitos universais. Em âmbito mundial, há medidas que asseguram a saúde do trabalhador, através do uso de EPI's.

Os dados apontaram fragilidade vivenciada pela equipe de enfermagem, uma vez que o profissional pensa ter conhecimento suficiente para cuidar de si e do outro, sem sofrer acidentes. Contudo, notou-se maior preocupação com a segurança pessoal e coletiva, no ambulatório de quimioterapia, por ser um serviço especializado em administração de Qt-An. Na unidade de clínica médica os dados desta pesquisa foram analisados e discutidos, onde chegou-se à conclusão de que é necessária uma intervenção eficiente para conscientizar seus profissionais frente aos riscos ocupacionais a que estão expostos.

Os resultados apontaram o conhecimento dos profissionais de enfermagem quanto ao significado da patologia em questão, aos riscos ocupacionais diante 
do tratamento ao paciente e à necessidade do uso de EPI's. Todavia, as respostas indicam a necessidade de investimento em aperfeiçoamento profissional, visando a educação permanente para melhor conscientização acerca do autocuidado e para refletir positivamente no cuidado do outro. As medidas de biossegurança devem estar presentes em todos os momentos do cuidado prestado pelo profissional de enfermagem. Para tanto, é necessário educar continuamente esses profissionais, realizando oficinas educativas, palestras e momentos de discussão sobre a importância desse tema.

\section{REFERÊNCIAS}

1.Gomez CM, Lacaz FAC. Saúde do trabalhador: novasvelhas questões. Ciênc saúde coletiva. 2005; 10:797-807. 2.Duran ECM, Robazzi MLCC, Marziale MHP. Conhecimento de enfermagem em saúde do trabalhador oriundo de dissertações e teses. Rev Gaúcha Enferm. 2007; 28:416-23.

3.Almeida ANG, Tipple AFV, Souza ACS, Brasileiro ME. Risco biológico entre os trabalhadores de enfermagem. Rev enferm UERJ. 2009; 17:595-600.

4.Alves SSM, Passos JP, Tocantins FR. Acidentes com perfurocortantes em trabalhadores de enfermagem: uma questão de biossegurança. Rev enferm UERJ. 2009; 17:373-7.

5.Silva LF, Reis PED. Avaliação do conhecimento da equipe de enfermagem sobre riscos ocupacionais na administração de quimioterápicos. Rev Bras Cancerol. 2010; 56:311-20.

6.Instituto Nacional do Câncer (Br). Ações de enfermagem para o controle do câncer: uma proposta de integração ensino-serviço. 3a ed. Rio de Janeiro: INCA; 2013.

7.Maia PG, Brito JC. Riscos relacionados à exposição de trabalhadores a quimioterápicos antineoplásicos: uma análise crítica da produção científica brasileira. Revista Tempus Actas de Saúde Coletiva. 2011; 5:51-65.

8.Gallas SR, Fontana RT. Biossegurança e a enfermagem nos cuidados clínicos: contribuições para a saúde do trabalhador. Rev Bras Enferm. 2010; 63:786-92.

9.Conselho Regional de Enfermagem. Estatística de profissionais cadastrados no COREn-SC. [citado em 06 nov 2013]. Disponível em http://www.coren-sc.org.br. 10.Neves HCC, Souza ACS, Barbosa JM, Ribeiro LCM, Tipple AFV, Alves SB, Suzuki K. O uso de equipamentos de proteção individual por profissionais em unidades de endoscopia. Rev enferm UERJ. 2010; 18:61-6.

11.Maia PG. A atividade da equipe de enfermagem e os riscos relacionados à exposição a quimioterápicos antineoplásicos no setor de oncologia de um hospital público do estado do Rio de Janeiro [dissertação de mestrado]. Rio de Janeiro: Escola Nacional de Saúde Pública Sergio Arouca; 2009.

12.Xelegati R, Robazzi MLCC, Marziale MHP, Haas VJ. Riscos ocupacionais químicos identificados por enfermeiros que trabalham em ambiente hospitalar. Rev Latino-Am Enfermagem. 2006; 14:214-9.

13.Universidade Federal de Santa Catarina. Comunicação de acidente de trabalho. [citado em 11 nov 2010]. Disponível em: http://www.dsst.ufsc.br/acidentes-de-trabalho. 14.Randon FR. Avaliação dos níveis de genotoxicidade e estresse oxidativo em manipuladores de quimioterápicos em serviços de oncologia [dissertação de mestrado]. Caxias do Sul (RS): Universidade de Caxias do Sul; 2006. 15.Valle ARMC, Moura MEB, Nunes BMVT, Figueiredo MLF. A biossegurança sob o olhar de enfermeiros. Rev enferm UERJ. 2012; 20: 361-7. 